\title{
Multimorbidity patterns in the elderly: a prospective cohort study with cluster analysis
}

\author{
Marina Guisado-Clavero 1,2,3, Albert Roso-Llorach ${ }^{1,2}$, Tomàs López-Jimenez ${ }^{1,2}$, Mariona Pons-Vigués ${ }^{1,2,4}$, \\ Quintí Foguet-Boreu ${ }^{1,2,5}$, Miguel Angel Muñoz $z^{2,3,6}$ and Concepción Violán 1,2*
}

\begin{abstract}
Background: Multimorbidity is the coexistence of more than two chronic diseases in the same individual; however, there is no consensus about the best definition. In addition, few studies have described the variability of multimorbidity patterns over time. The aim of this study was to identify multimorbidity patterns and their variability over a 6 -year period in patients older than 65 years attended in primary health care.

Methods: A cohort study with yearly cross-sectional analysis of electronic health records from 50 primary health care centres in Barcelona. Selected patients had multimorbidity and were 65 years of age or older in 2009. Diagnoses (International Classification of Primary Care, second edition) were extracted using O'Halloran criteria for chronic diseases. Multimorbidity patterns were identified using two steps: 1) multiple correspondence analysis and 2) k-means clustering. Analysis was stratified by sex and age group (65-79 and $\geq 80$ years) at the beginning of the study period.

Results: Analysis of 2009 electronic health records from 190,108 patients with multimorbidity (59.8\% women) found a mean age of 71.8 for the 65-79 age group and 84.16 years for those over 80 (Standard Deviation [SD] 4.35 and 3.46, respectively); the median number of chronic diseases was seven (Interquartil range [IQR] 5-10). We obtained 6 clusters of multimorbidity patterns (1 nonspecific and 5 specifics) in each group, being the specific ones: Musculoskeletal, Endocrine-metabolic, Digestive/Digestive-respiratory, Neurological, and Cardiovascular patterns. A minimum of 42. $5 \%$ of the sample remained in the same pattern at the end of the study, reflecting the stability of these patterns.

Conclusions: This study identified six multimorbidity patterns per each group, one nonnspecific pattern and five of them with a specific pattern related to an organic system. The multimorbidity patterns obtained had similar characteristics throughout the study period. These data are useful to improve clinical management of each specific subgroup of patients showing a particular multimorbidity pattern.
\end{abstract}

Keywords: Multimorbidity, Chronic disease, Ageing, Primary health care, Cluster analysis, Electronic health record

\section{Background}

Multimorbidity is defined as the coexistence of two or more chronic diseases $[1,2]$. Although overall life expectancy and healthy life years have increased worldwide, quality of life and functional capacity has worsened [3] due to the chronic conditions strongly related to aging. Some studies predict a rise in prevalence of these

\footnotetext{
* Correspondence: cviolan@idiapjgol.org

${ }^{1}$ Institut Universitari d'Investigació en Atenció Primària Jordi Gol (IDIAP Jordi Gol), Gran Via Corts Catalanes, 587 àtic, 08007 Barcelona, Spain

2Universitat Autònoma de Barcelona, Campus de la UAB, Plaça Cívica, 08193,

Bellaterra (Cerdanyola del Vallès), Barcelona, Spain

Full list of author information is available at the end of the article
}

conditions [4]; population multimorbidity prevalence currently ranges from $12.9 \%$ to $95.1 \%$ [5]. In addition, rates of hospitalization and treatment for people with chronic diseases have soared; consequently, a growth in the burden of disease on health systems is assumed in general, and in primary health care in particular [3].

Although life expectancy has increased in the last century [3], research on multimorbidity has been limited and has focused on describing prevalence, estimating severity, and assessing quality of life [6, 7].

In clinical practice, individual patients often present with a collection of chronic diseases which may or may 
not have a common aetiology, but which require greatly differing and often incompatible management. Prevalence studies, mostly with transversal designs, have identified multimorbidity patterns in patients older than 65 years, but few prospective longitudinal studies have been published and none of them have analysed a period longer than 4 years [5]. With better knowledge about the evolution of multimorbidity patterns, the joint management of several chronic diseases simultaneously could be more effective.

On the other hand, most of the published studies considered diseases, not individuals, as the variable of analysis in assessing multimorbidity patterns. This inhibits an exploration of multimorbidity patterns that takes into account their trajectories and evolution along the individual's lifetime.

Finally, no consensus has been established about a standard model to determine multimorbidity patterns. Published studies differ in the variables included, such as the unit of analysis selected (patients versus diseases), the statistical method for grouping diseases (factor analysis vs. cluster analysis), diseases included (chronic and/ or acute), and number of diseases considered $[8,9]$. Nevertheless, non-hierarchical cluster analysis assigns patients into a specified number of clusters [10]. The results are less susceptible to outliers in the data, the influence of the distance measure chosen, or the inclusion of inappropriate or irrelevant variables. Some nonhierarchical cluster analysis methods, like k-means, use algorithms that do not need a distance matrix and can analyse extremely large data sets [10-12].

The aim of this study was to identify multimorbidity patterns over a six-year study period in electronic health records from a Mediterranean urban population older than 65 years and with multimorbidity, attended in primary health care centres in Barcelona (Spain).

\section{Methods}

\section{Design, setting, and study population}

A cohort study with a cross-sectional analysis was carried out in each year of the study period, from 2009 to 2014, in Barcelona, Catalonia (Spain), a city of Mediterranean region with $1,619,337$ inhabitants (31/12/2009) [13]. The Spanish National Health Service provides universal coverage, financed mainly by tax revenue. The Catalan Health Institute (CHI) manages 50 primary health care centres (PHCs) in Barcelona that represent $74 \%$ of the population [14]. The CHI's Information System for Research in Primary Care (SIDIAP) contains the clinical information as electronic health records (EHR) recorded by its PHCs since 2006 [15-17].

Inclusion criteria were 65-94 years of age on 31 December 2009 and at least one PHC visit during the 6year study period. From the initial sample of 206,146
(Fig. 1), we excluded people who moved or otherwise sought care outside the $\mathrm{CHI}$ system. The only reason to exit the cohort was death $(n=24,013)$, and no new participants were introduced during the study period.

Prevalence of individual conditions varies with age, as does multimorbidity and disease patterns. In order to obtain a more homogenous sample in terms of multimorbidity, we focused on patients from Barcelona city with multimorbidity, defined as 2 or more diagnoses of chronic disease active as of 31 December 2009. We obtained information on that population during 6 years and analysed the data 6 times at cross-sectional time points, every December from 2009 to 2014. However, mortality data were obtained 5 times, from 2010 to 2014.

\section{Coding and selection of diseases}

Diseases are coded in SIDIAP using International Classification of Diseases version 10 (ICD-10). We mapped ICD-10 codes to International Classification of Primary Care, second edition (ICPC-2) codes in order to select chronic diseases by O'Halloran criteria [18] based on the ICPC-2. We only considered chronic diseases with a prevalence over $1 \%$ to avoid spurious associations and obtain epidemiologically coherent patterns. Chronic diseases were coded as a dichotomous variable.

\section{Variables}

The unit of measurement was the diagnosis (values: 1 for present, 0 for absent). Other variables recorded for each patient were the following: number of different diseases (chronic diseases active on 31 December each year), age groups in 2009 (65-79; $\geq 80$ ), and sex (women, men).

\section{Statistical analysis}

Data access: Data were obtained from SIDIAP after the study was authorized. All authors were granted access to the database. No missing values were handled, as sex and age were universally recorded, so there were no missing values and no missing data were imputed. Wrong codes for sex-specific diagnoses and diagnoses with inconsistent dates were excluded.

\section{Descriptive analysis}

Analyses were stratified by sex and age. Descriptive statistics were used to summarize overall information. Categorical variables were expressed as frequencies (percentage) and continuous as mean (Standard deviation, SD) or median (interquartile range, IQR). Chisquare test and Mann-Whitney test were used to assess differences between age groups by sex.

Prevalence of each chronic disease was calculated for each year in order to study the evolution over time. Multimorbidity patterns were identified using two steps: 1) multiple correspondence analysis (MCA) and 2) k- 


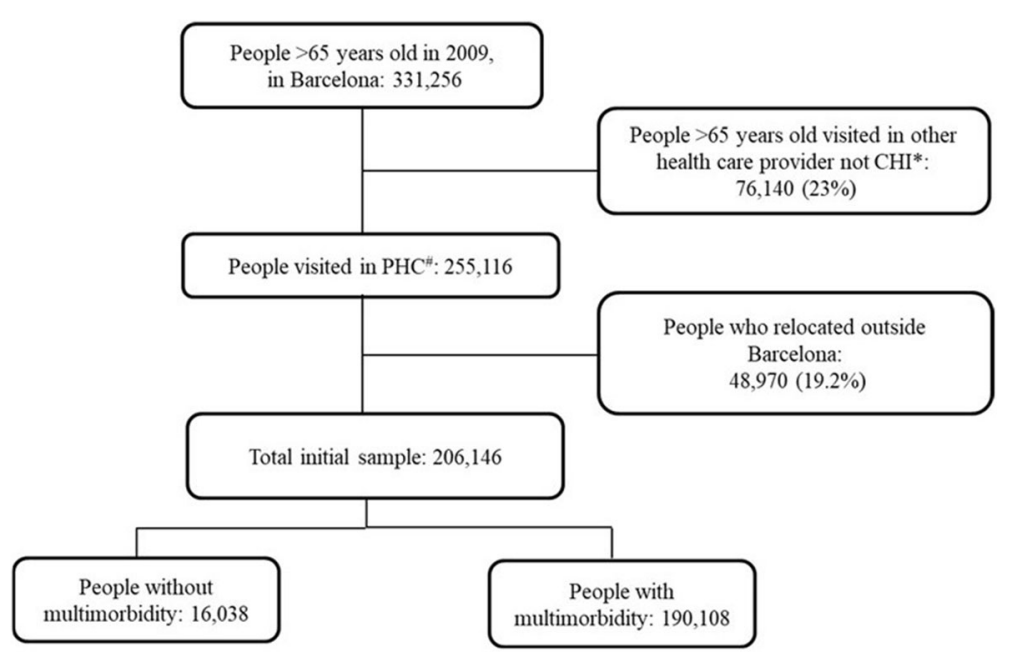

Fig. 1 Flow chart of the study

means clustering. For every year of study (2009-14), MCA and k-means analysis included only those individuals that were alive as of 31 December each year.

\section{Multiple correspondence analysis}

This data analysis technique for nominal categorical data was used to detect and represent underlying structures in the data set. The MCA method allows representation in a multidimensional space of relationships between a set of dichotomous or categorical variables, in our case diagnoses, that would otherwise be difficult to observe in contingency tables and to show groups of patients with the same characteristics $[19,20]$. MCA also allows the direct representation of patients as points (coordinates) in geometric space, transforming the original binary data to continuous data. The MCA analysis was based on the indicator matrix. Optimal number of dimensions extracted and percentages of inertia were determined by scree plot.

\section{k-means clustering}

From the geometric space created in MCA, patients were classified in clusters according to proximity criteria using the k-means algorithm with random initial centroids. Clusters centres were obtained for each cluster. Optimal number of clusters ( $\mathrm{k}$ ) was assessed according to Calinski Harabaz criteria, using 100 iterations. The optimal number of clusters is the solution with the highest Calinski-Harabaz index value. To assess internal cluster quality, cluster stability of the optimal solution was computed using Jaccard bootstrap values with 100 runs [10]. "Highly stable" clusters should yield average Jaccard similarities of 0.85 and above.

\section{Multimorbidity patterns}

To describe multimorbidity patterns, frequencies and percentage of diseases in each cluster were calculated. Observed/expected (O/E) ratios were obtained by dividing disease prevalence in the cluster by disease prevalence in each age group, by sex. To define a specific pattern, we considered those diseases with an intracluster prevalence $\geq 20 \%$ and an over-expression with $\mathrm{O} /$ $E$ ratio $\geq 2$ [21]. The names of patterns are related to the main system affected in each cluster.

Descriptive statistics of age and number of diagnoses per each cluster were also obtained. Clinical criteria were used to evaluate the consistency and utility of the final cluster solution, based on clusters previously described in the literature and a consensus opinion drawn from the clinical experience of the research team (3 family physicians and 2 epidemiologists engaged in daily patient care). Stability in the patterns was considered as the number of persons staying in the same pattern in 2014, as well as the percentage of people who remained in the same pattern at the end of the study compared to 2009.

The consistency of multimorbidity patterns was established by analysing the number (percentage) of people who remained stable within the cluster during the study period.

The analyses were carried out using SPSS for Windows, version 18 (SPSS Inc., Chicago, IL, USA) and R version 3.3.1, procedures FactorMineR, $\mathrm{fpc}$, and vegan $(\mathrm{R}$ Foundation for Statistical Computing, Vienna, Austria).

\section{Results}

Out of 206,146 persons analysed at the beginning of the study in 2009, 190,108 (92.2\%) fulfilled multimorbidity criteria (Fig. 1) and 59.8\% were women. The mean age at the beginning of the study was 71.8 (SD 4.35) years for 
the group 65-79 years old, and 84.2 years (SD 3.46) for the group over 80 . In $2009,31.2 \%$ to $39.1 \%$ of the population had fewer than 5 chronic diseases, while $40.2 \%$ to $42.3 \%$ had 6 to 9 diseases and $20.7 \%$ to $28.2 \%$ had received more than 10 diagnoses. The median number of diseases was 7 (IQR 5-10) for women and for men older than 80 years; the younger men (aged 65-79 years) had a median of 6 diseases (IQR 4-9) (Table 1).

\section{Chronic diseases prevalence}

Hypertension, uncomplicated was the most prevalent chronic disease in all groups over the period of time studied, followed by Lipid disorder. In the group aged 65-79 years, uncomplicated hypertension affected 69\% of women and $68 \%$ of men in 2009 , and lipid disorder affected $57.7 \%$ and $49.4 \%$, respectively. Other prevalent diagnoses for women in this age group in 2009 were Osteoporosis (32.6\%), Obesity (29.2\%), and Depressive disorder (27.3\%); among men, ageing-related diseases were prevalent, including Benign prostatic hypertrophy (41.6\%), Cataracts (21.4\%), and Diabetes, non-insulindependent (30.8\%). The top 10 chronic diseases for women and men throughout the study period are shown in Fig. 2. Few changes in prevalence were observed over the 6 years analysed.

\section{K-means clustering}

Using the Calinski criterion, six clusters were considered as the optimal solution for both age and sex strata. Average Jaccard bootstrap values for both women and men were 0.85 and above.

\section{Multimorbidity patterns}

For each of the four groups studied (two age groups of men and women), 6 clusters were identified using the kmeans method. The first pattern, formed by only the most prevalent diseases, was named the "nonspecific" pattern; the remaining 5 patterns were specific to Musculoskeletal, Endocrine-metabolic, Digestive/digestive-respiratory, Neuropsychiatric, and Cardiovascular diseases, in decreasing order depending on the percentage of the population included [see Additional files 1, 2].

The first cluster had the largest percentage of the sample, both women and men: 35.6 and $36.7 \%$ of those aged $65-79$ years, $34.3-34.1 \%$ of those aged 80 and older respectively [see Additional files 1-4]. For women, the

Table 1 Number of diseases, stratified by sex and age group

\begin{tabular}{|c|c|c|c|c|}
\hline \multicolumn{5}{|l|}{ Patients included in the analysis in $2009, N=190,108$} \\
\hline & \multicolumn{2}{|l|}{ Women } & \multicolumn{2}{|l|}{ Men } \\
\hline & $65-79$ years & $\geq 80$ years & $65-79$ years & $\geq 80$ years \\
\hline Total, $n(\%)$ & $80,208(42.2)$ & $33,442(17.6)$ & $59,331(31.2)$ & $17,127(9.0)$ \\
\hline \multicolumn{5}{|l|}{ Number of diagnoses ${ }^{*}, n(\%)$} \\
\hline 2 & $4249(5.3)$ & $1595(4.8)$ & $3770(6.4)$ & $782(4.6)$ \\
\hline [3-5] & $22,491(28.1)$ & $8688(26.0)$ & $19,429(32.7)$ & $4534(26.5)$ \\
\hline [6-9] & $32,663(40.7)$ & $13,732(41.0)$ & $23,846(40.2)$ & $7252(42.3)$ \\
\hline$\geq 10$ & $20,805(25.9)$ & $9427(28.2)$ & $12,286(20.7)$ & $4559(26.6)$ \\
\hline Median number of diagnoses (IQR) ${ }^{* *}$ & $7(5-10)$ & $7(5-10)$ & $6(4-9)$ & $7(5-10)$ \\
\hline Number of diagnoses included in cluster analysis ${ }^{* * *}$ & 83 & 85 & 84 & 85 \\
\hline \multicolumn{5}{|l|}{ Patients included in the analysis in $2014, N=166,095$} \\
\hline & Women & & Men & \\
\hline & 70-79 years & $\geq 80$ years & 70-79 years & $\geq 80$ years \\
\hline Total $n(\%)$ & $51,835(31.2)$ & $49,940(30.1)$ & $38,127(22.9)$ & $26,193(15.8)$ \\
\hline \multicolumn{5}{|l|}{ Number of diagnoses ${ }^{*}, n(\%)$} \\
\hline 2 & $3220(6.2)$ & $2026(4.1)$ & $2811(7.4)$ & $1125(4.3)$ \\
\hline$[3-5]$ & $16,662(32.1)$ & $12,144(24.3)$ & $13,879(36.4)$ & $6818(26.0)$ \\
\hline [6-9] & $21,334(41.2)$ & $21,316(42.7)$ & $15,119(39.6)$ & $11,333(43.3)$ \\
\hline$\geq 10$ & $10,619(20.5)$ & $14,454(28.9)$ & $6318(16.6)$ & $6917(26.4)$ \\
\hline Median number of diagnoses $(\mathrm{IQR})^{* *}$ & $6(4-9)$ & $7(5-10)$ & $6(4-8)$ & $7(5-10)$ \\
\hline Number of diagnoses included in cluster analysis ${ }^{* *}$ & 80 & 84 & 80 & 82 \\
\hline
\end{tabular}

Abbreviations: IQR Inter-quartile range

"Chi-square test; all $p$-values $<0.001$

*Mann-Whitney test; $P<0.001$

${ }^{* * *}$ Chronic diseases with $>1 \%$ prevalence 


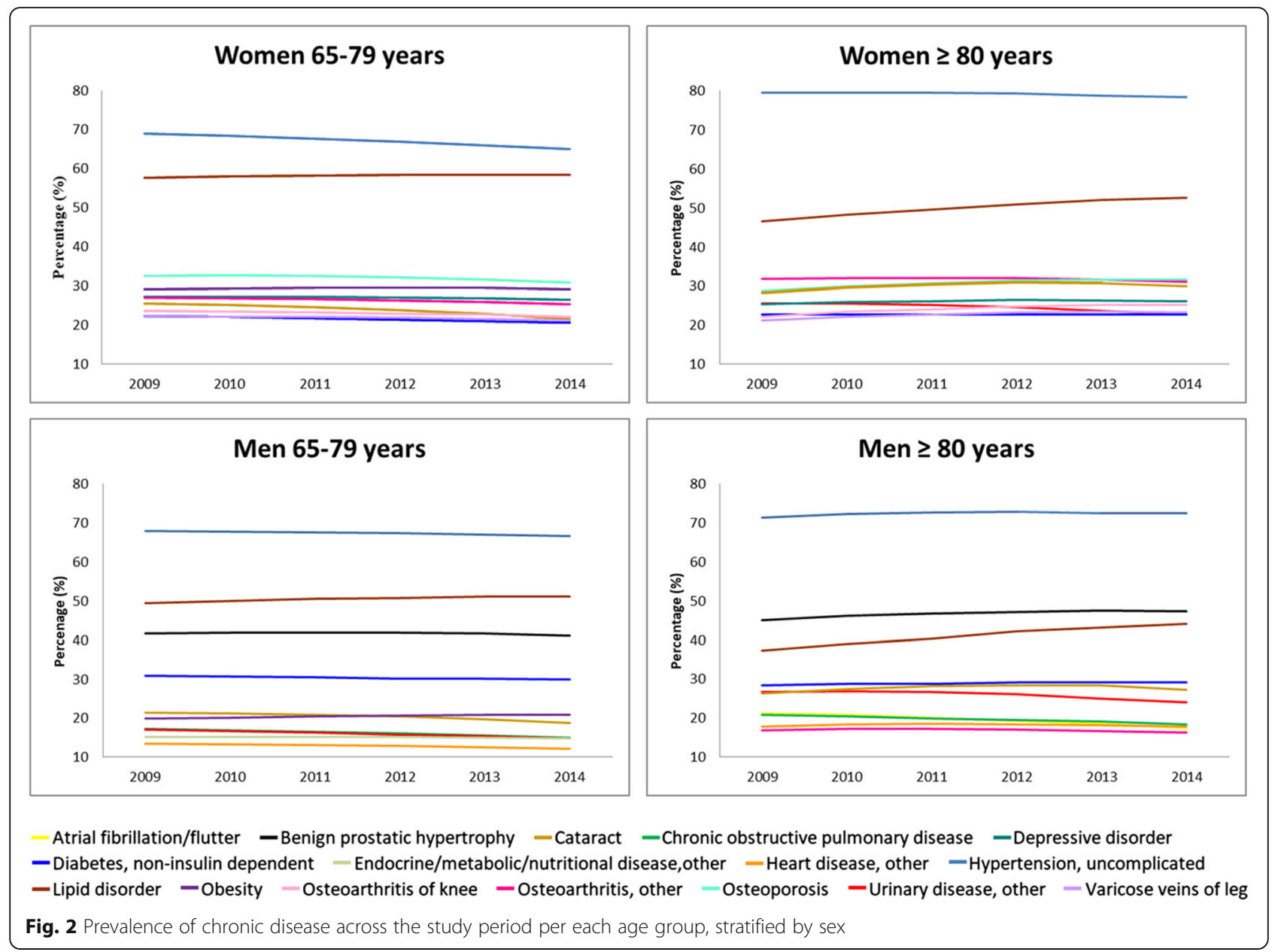

top 3 diagnoses throughout the study period were Hypertension, uncomplicated; Lipid disorder; and Osteoporosis. In the older group, Osteoarthritis, other was added to the list for the first year and Cataract for the other 5 years analysed [see Additional files 1-3].Similarly for men, three diseases predominated in the Nonspecific pattern throughout the study period: Hypertension, uncomplicated; Lipid disorder, and Benign prostatic hypertrophy. In older men, these diseases were joined by Diabetes, non-insulin dependent in the first year, adding Cataract in the remaining 5 years [see Additional files 2, 4]. There was no over-represented disease in these groups $(\mathrm{O} / \mathrm{E}$ ratio $\geq 2)$.

Few variations were detected in terms of prevalence and $\mathrm{O} / \mathrm{E}$ ratios for the elements of a specific cluster, as shown in the example presented in Tables 2 and 3. A pattern observed in women aged 65-79 years was labelled the Neuropsychiatric pattern (Table 2). Some neurological diseases were over-represented in 2009, such as Dementia (O/E ratio 5.98) or Stroke/cerebrovascular accident $(\mathrm{O} / \mathrm{E}$ ratio 4.81), with a prevalence $\geq 20 \%$. Other over-represented diseases $(\mathrm{O} / \mathrm{E}$ ratio $\geq 2)$ had a prevalence $<20 \%$ and bear little relation to the main system affected, such as Ischaemic heart disease without angina $(\mathrm{O} / \mathrm{E}$ ratio 4.27 , prevalence of $13.9 \%)$ or Atherosclerosis/peripheral vascular disease $(\mathrm{O} / \mathrm{E}$ ratio 3.08, prevalence of 9.6\%). A large number of patients (in the Table 2, 42.5\% of women aged 65-79 years) stayed in the same pattern from baseline until the end of the study period. The rest of these percentages are presented in [see Additional files 1, 2].

Table 3 shows men aged 65-79 years with the Neuropsychiatric pattern, containing almost the same diseases as the homologous pattern in women. Differences between the patterns are mainly sex-related diseases such as Benign prostatic hypertrophy.

Following the same method as these two examples, it can be observed that chronic diseases included in each pattern at the beginning of the sample mostly persisted throughout the 6 years analysed. Some variations were observed, such as chronic disease leaving the pattern when it did not meet the inclusion criteria, sometimes only by a few decimal points that decided whether a disease remained in a pattern or not [see Additional files 1-4]. 
Table 2 Example of multimorbidity pattern: neuropsychiatric pattern considering observed/expected ratio in one cluster across women aged $65-79$ years

\begin{tabular}{|c|c|c|c|c|c|c|c|c|c|c|c|c|c|}
\hline \multicolumn{2}{|c|}{ Neuropsychiatric pattern } & \multicolumn{2}{|l|}{2009} & \multicolumn{2}{|l|}{2010} & \multicolumn{2}{|l|}{2011} & \multicolumn{2}{|l|}{2012} & \multicolumn{2}{|l|}{2013} & \multicolumn{2}{|l|}{2014} \\
\hline \multicolumn{2}{|c|}{ Number of persons in the cluster } & \multicolumn{2}{|c|}{$n=8242$} & \multicolumn{2}{|c|}{$n=7493$} & \multicolumn{2}{|c|}{$n=6770$} & \multicolumn{2}{|c|}{$n=6042$} & \multicolumn{2}{|c|}{$n=5360$} & \multicolumn{2}{|c|}{$n=4688$} \\
\hline \multicolumn{2}{|c|}{ Persons stable in the pattern. $n(\%)$} & \multicolumn{2}{|c|}{$8242^{a}(100)$} & \multicolumn{2}{|c|}{$7210^{\mathrm{a}}(87.5)$} & \multicolumn{2}{|c|}{$6297^{\mathrm{a}}(76.4)$} & \multicolumn{2}{|c|}{$5386^{\mathrm{a}}(65.4)$} & \multicolumn{2}{|c|}{$4536^{\mathrm{a}}(55.0)$} & \multicolumn{2}{|c|}{$3503^{\mathrm{a}}(42.5)$} \\
\hline ICPC2 & Diagnoses & $\begin{array}{l}\text { Prev }^{\mathrm{b}} \\
(\%)\end{array}$ & $\underset{r^{c}}{\mathrm{O} / \mathrm{E}}$ & $\begin{array}{l}\text { Prev }^{\mathrm{b}} \\
(\%)\end{array}$ & $\begin{array}{l}\mathrm{O} / \mathrm{E} \\
\mathrm{r}^{\mathrm{c}}\end{array}$ & $\begin{array}{l}\text { Prev }^{\mathrm{b}} \\
(\%)\end{array}$ & $\underset{r^{c}}{\mathrm{O} / \mathrm{E}}$ & $\begin{array}{l}\text { Prev }^{\mathrm{b}} \\
(\%)\end{array}$ & $\begin{array}{l}\mathrm{O} / \mathrm{E} \\
\mathrm{r}^{\mathrm{c}}\end{array}$ & $\begin{array}{l}\text { Prev }^{\mathrm{b}} \\
(\%)\end{array}$ & $\begin{array}{l}\mathrm{O} / \mathrm{E} \\
\mathrm{r}^{\mathrm{c}}\end{array}$ & $\begin{array}{l}\text { Prev }^{\mathrm{b}} \\
(\%)\end{array}$ & $\underset{r^{c}}{\mathrm{O} / \mathrm{E}}$ \\
\hline K86 & Hypertension, uncomplicated & 70.4 & 1.02 & 69.8 & 1.02 & 68.7 & 1.01 & 68.6 & 1.03 & 68.6 & 1.04 & 70.8 & 1.09 \\
\hline T93 & Lipid disorder & 67.6 & 1.17 & 68.4 & 1.18 & 68.1 & 1.17 & 67.9 & 1.16 & 68.4 & 1.17 & 70.6 & 1.21 \\
\hline P76 & Depressive disorder & 53.5 & 1.96 & 53.7 & 1.97 & 53.1 & 1.95 & 53.4 & 1.97 & 51.3 & 1.90 & 49.2 & 1.86 \\
\hline L95 & Osteoporosis & 39.5 & 1.21 & 39.9 & 1.22 & 40.0 & 1.23 & 39.3 & 1.22 & 39.2 & 1.24 & 39.2 & 1.27 \\
\hline P70 & Dementia & 32.4 & 5.98 & 30.8 & 6.31 & 30.1 & 6.79 & 27.9 & 7.05 & 25.4 & 7.33 & 21.0 & 6.94 \\
\hline L91 & Osteoarthritis. other & 31.8 & 1.18 & 31.1 & 1.16 & 30.1 & 1.13 & 30.4 & 1.16 & 29.5 & 1.14 & 29.3 & 1.15 \\
\hline T90 & Diabetes. non-insulin dependent & 31.0 & 1.38 & 30.9 & 1.40 & 30.6 & 1.40 & 32.8 & 1.52 & 33.7 & 1.60 & 37.4 & 1.81 \\
\hline F92 & Cataract & 29.4 & 1.15 & 29.2 & 1.16 & 29.1 & 1.18 & 29.2 & 1.22 & 28.2 & 1.23 & 26.4 & 1.22 \\
\hline K90 & Stroke/cerebrovascular accident & 23.5 & 4.81 & 23.1 & 4.94 & 22.4 & 5.02 & 21.5 & 5.11 & 20.2 & 5.08 & 16.9 & 4.50 \\
\hline P71 & Organic psychosis, other & 16.0 & 5.72 & 16.2 & 6.06 & 16.0 & 6.25 & 15.8 & 6.46 & 15.5 & 6.61 & 14.6 & 6.66 \\
\hline K76 & $\begin{array}{l}\text { Ischaemic heart disease without } \\
\text { angina }\end{array}$ & 13.9 & 4.27 & 13.9 & 4.47 & 13.6 & 4.57 & 14.2 & 5.04 & 15.4 & 5.68 & 17.9 & 7.00 \\
\hline K91 & Cerebrovascular disease & 12.9 & 6.05 & 12.5 & 6.11 & 12.4 & 6.23 & 12.4 & 6.33 & 12.4 & 6.55 & 11.5 & 6.36 \\
\hline N87 & Parkinsonism & 12.8 & 6.62 & 11.9 & 6.51 & 12.2 & 7.00 & 11.6 & 7.12 & 11.7 & 7.47 & 11.1 & 7.72 \\
\hline N99 & Neurological disease, other & 11.6 & 4.63 & 11.9 & 4.85 & 11.8 & 4.88 & 11.8 & 5.00 & 11.0 & 4.78 & 10.7 & 4.86 \\
\hline K92 & $\begin{array}{l}\text { Atherosclerosis/ peripheral vascular } \\
\text { disease }\end{array}$ & 9.6 & 3.08 & 9.6 & 3.17 & 9.6 & 3.30 & 9.6 & 3.45 & 9.4 & 3.47 & 11.4 & 4.36 \\
\hline K89 & Transient cerebral ischaemia & 9.3 & 3.67 & 9.1 & 3.74 & 8.6 & 3.69 & 8.7 & 3.90 & 8.0 & 3.77 & 6.9 & 3.45 \\
\hline K74 & Ischaemic heart disease with angina & 8.7 & 3.84 & 8.9 & 3.97 & 8.6 & 4.03 & 9.0 & 4.48 & 9.1 & 4.81 & 11.4 & 6.54 \\
\hline K75 & Acute myocardial infarction & 7.9 & 4.79 & 8.0 & 5.11 & 7.7 & 5.16 & 7.8 & 5.60 & 8.7 & 6.53 & 9.6 & 7.61 \\
\hline N88 & Epilepsy & 6.4 & 5.83 & 6.5 & 6.08 & 6.9 & 6.45 & 6.6 & 6.27 & 6.4 & 6.28 & - & - \\
\hline B81 & Anaemia. Vit B12/folate deficiency & 4.0 & 2.83 & 3.7 & 2.74 & 3.6 & 2.78 & 3.5 & 2.86 & 3.5 & 3.09 & 2.9 & 2.80 \\
\hline F83 & Retinopathy & - & - & 5.5 & 2.12 & 5.9 & 2.32 & 7.2 & 2.87 & 7.2 & 2.96 & 9.8 & 4.13 \\
\hline N94 & Peripheral neuritis/neuropathy & - & - & - & - & - & - & 5.3 & 2.15 & 5.5 & 2.28 & 7.0 & 2.98 \\
\hline T82 & Obesity & - & - & - & - & - & - & 21.1 & 0.72 & 21.8 & 0.74 & 23.0 & 0.79 \\
\hline B82 & Anaemia. other/unspecified & - & - & - & - & - & - & - & - & 13.1 & 2.09 & 13.9 & 2.46 \\
\hline F84 & Macular degeneration & - & - & - & - & - & - & - & - & - & - & 3.2 & 2.24 \\
\hline
\end{tabular}

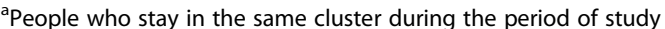

${ }^{\mathrm{b}}$ Prev: prevalence

${ }^{\mathrm{C}} \mathrm{O} / \mathrm{E} \mathrm{r}$ : observed/expected ratio. Italic numbers indicate $\mathrm{O} / \mathrm{E} \mathrm{r} \geq 2$

Among women aged 80 and older, as in the younger group, we defined six clusters (Nonspecific and 5 specific multimorbidity patterns) with the same names, even if the diseases varied, because the main system affected was the same. The Muskuloskeletal, Endocrine-metabolic, Digestive and Cardiovascular patterns showed changes in 1 or 2 diseases, but the Neuropsychiatric pattern had added 4 diseases to the cluster by the end of the study period [see Additional file 3].

Several differences were observed in the older group of men, as well. First, the Endocrine-metabolic pattern in this age group was defined by diseases localized in the Cardiovascular patterns in men aged 65-79 years.
Secondly, the Digestive pattern incorporated respiratory diseases, becoming the Digestive-respiratory pattern (as in the last year analysed in men 65-79 years), composed of 9 more chronic diseases than the Digestive pattern. Thirdly, the Neuropsychiatric and Cardiovascular patterns lost some diseases. Finally, no important changes were found in the Musculoskeletal pattern [see Additional file 4].

Furthermore, the percentage of patients whose multimorbidity pattern remained stable exceeded $42.5 \%$ for all patterns per each sex and age group. The Nonspecific patterns had the highest values for stability at the end of the period for all groups except men aged 
Table 3 Example of multimorbidity pattern: neuropsychiatric pattern considering observed/expected ratio in one cluster across men aged 65-79 years

\begin{tabular}{|c|c|c|c|c|c|c|c|c|c|c|c|c|c|}
\hline \multicolumn{2}{|c|}{ Neuropsychiatric pattern } & \multicolumn{2}{|l|}{2009} & \multicolumn{2}{|l|}{2010} & \multicolumn{2}{|l|}{2011} & \multicolumn{2}{|l|}{2012} & \multicolumn{2}{|l|}{2013} & \multicolumn{2}{|l|}{2014} \\
\hline \multicolumn{2}{|c|}{ Number of persons in the cluster } & \multicolumn{2}{|c|}{$n=5956$} & \multicolumn{2}{|c|}{$n=5644$} & \multicolumn{2}{|c|}{$n=5080$} & \multicolumn{2}{|c|}{$n=4483$} & \multicolumn{2}{|c|}{$n=3819$} & \multicolumn{2}{|c|}{$n=3274$} \\
\hline \multicolumn{2}{|c|}{ Patients stable in the pattern, $n(\%)$} & \multicolumn{2}{|c|}{$5956^{\mathrm{a}}(100)$} & \multicolumn{2}{|c|}{$5013^{\mathrm{a}}(84.2)$} & \multicolumn{2}{|c|}{$4205^{\mathrm{a}}(70.6)$} & \multicolumn{2}{|c|}{$3757^{\mathrm{a}}(63.1)$} & \multicolumn{2}{|c|}{$3214^{\mathrm{a}}(54.0)$} & \multicolumn{2}{|c|}{$2741^{\mathrm{a}}(46.0)$} \\
\hline ICPC2 & Diagnosis & $\begin{array}{l}\text { Prev }^{\mathrm{b}} \\
(\%)\end{array}$ & $\begin{array}{l}\mathrm{O} / \mathrm{E} \\
\mathrm{r}^{\mathrm{c}}\end{array}$ & $\begin{array}{l}\text { Prev }^{\mathrm{b}} \\
(\%)\end{array}$ & $\underset{r^{c}}{\mathrm{O} / \mathrm{E}}$ & $\begin{array}{l}\text { Prev }^{\mathrm{b}} \\
(\%)\end{array}$ & $\begin{array}{l}\mathrm{O} / \mathrm{E} \\
\mathrm{r}^{\mathrm{c}}\end{array}$ & $\begin{array}{l}\text { Prev }^{\mathrm{b}} \\
(\%)\end{array}$ & $\begin{array}{l}\mathrm{O} / \mathrm{E} \\
\mathrm{r}^{\mathrm{c}}\end{array}$ & $\begin{array}{l}\text { Prev }^{\mathrm{b}} \\
(\%)\end{array}$ & $\underset{r^{c}}{\mathrm{O} / \mathrm{E}}$ & $\begin{array}{l}\text { Prev }^{b} \\
(\%)\end{array}$ & $\begin{array}{l}\mathrm{O} / \mathrm{E} \\
\mathrm{r}^{\mathrm{c}}\end{array}$ \\
\hline K86 & Hypertension, uncomplicated & 67.3 & 0.99 & 70.0 & 1.03 & 71.0 & 1.05 & 68.9 & 1.02 & 68.1 & 1.02 & 66.2 & 0.99 \\
\hline T93 & Lipid disorder & 54.5 & 1.10 & 58.6 & 1.17 & 60.6 & 1.20 & 59.9 & 1.18 & 60.3 & 1.18 & 58.7 & 1.15 \\
\hline Y85 & Benign prostatic hypertrophy & 45.7 & 1.10 & 43.9 & 1.05 & 42.4 & 1.01 & 42.4 & 1.02 & 42.9 & 1.03 & 44.5 & 1.08 \\
\hline T90 & Diabetes, non-insulin dependent & 37.8 & 1.23 & 42.5 & 1.39 & 45.5 & 1.49 & 43.2 & 1.43 & 42.1 & 1.40 & 38.2 & 1.28 \\
\hline K90 & Stroke/cerebrovascular accident & 34.9 & 4.31 & 35.4 & 4.56 & 35.5 & 4.73 & 35.0 & 4.86 & 35.3 & 5.11 & 35.5 & 5.34 \\
\hline P76 & Depressive disorder & 34.1 & 3.11 & 32.1 & 2.95 & 30.4 & 2.83 & 31.4 & 2.94 & 31.9 & 3.04 & 32.3 & 3.19 \\
\hline P70 & Dementia & 28.2 & 6.56 & 24.3 & 6.24 & 22.2 & 6.32 & 22.2 & 6.84 & 21.5 & 7.52 & 21.7 & 8.73 \\
\hline F92 & Cataract & 23.8 & 1.11 & 24.1 & 1.14 & 24.3 & 1.16 & 23.4 & 1.14 & 23.7 & 1.20 & 21.2 & 1.13 \\
\hline K76 & $\begin{array}{l}\text { Ischaemic heart disease without } \\
\text { angina }\end{array}$ & 21.5 & 2.12 & 24.0 & 2.42 & 24.4 & 2.52 & 24.4 & 2.57 & 22.5 & 2.44 & 20.0 & 2.23 \\
\hline K92 & $\begin{array}{l}\text { Atherosclerosis/peripheral vascular } \\
\text { disease }\end{array}$ & 19.6 & 2.16 & 23.4 & 2.63 & 25.2 & 2.92 & 23.7 & 2.83 & 22.6 & 2.78 & 20.3 & 2.60 \\
\hline K91 & Cerebrovascular disease & 19.4 & 5.84 & 20.1 & 6.08 & 20.9 & 6.46 & 20.8 & 6.57 & 21.4 & 6.90 & 21.2 & 7.07 \\
\hline N87 & Parkinsonism & 17.3 & 6.74 & 15.0 & 6.14 & 13.6 & 5.88 & 13.6 & 6.12 & 13.9 & 6.66 & 14.2 & 7.10 \\
\hline K75 & Acute myocardial infarction & 13.5 & 2.06 & 15.8 & 2.47 & 15.6 & 2.50 & 15.8 & 2.62 & 14.9 & 2.52 & 12.8 & 2.28 \\
\hline N99 & Neurological disease, other & 13.5 & 5.08 & 13.0 & 4.96 & 12.8 & 4.98 & 13.3 & 5.24 & 13.9 & 5.65 & 14.7 & 6.22 \\
\hline P71 & Organic psychosis, other & 13.5 & 6.13 & 12.3 & 5.77 & 11.7 & 5.66 & 11.4 & 5.76 & 12.0 & 6.35 & 12.6 & 6.99 \\
\hline K89 & Transient cerebral ischaemia & 11.1 & 3.58 & 10.6 & 3.52 & 10.3 & 3.54 & 9.8 & 3.50 & 9.7 & 3.63 & 9.0 & 3.62 \\
\hline K74 & Ischaemic heart disease with angina & 10.3 & 2.35 & 11.1 & 2.58 & 11.3 & 2.71 & 11.9 & 2.93 & 11.1 & 2.79 & 9.7 & 2.50 \\
\hline N88 & Epilepsy & 8.2 & 6.13 & 7.4 & 5.61 & 7.1 & 5.43 & 7.8 & 6.06 & 8.5 & 6.69 & 9.1 & 7.33 \\
\hline $\mathrm{A} 04$ & Weakness/tiredness. General & 6.4 & 2.63 & 6.1 & 2.50 & 6.0 & 2.53 & 5.7 & 2.51 & 5.9 & 2.83 & 5.7 & 3.13 \\
\hline B81 & Anaemia. Vit B12/folate deficiency & 4.0 & 2.63 & 3.9 & 2.65 & 3.9 & 2.79 & 3.1 & 2.39 & 2.8 & 2.20 & 2.6 & 2.12 \\
\hline B82 & Anaemia. other/unspecified & - & - & 14.5 & 2.01 & 14.8 & 2.13 & 13.7 & 2.08 & - & - & - & - \\
\hline F83 & Retinopathy & - & - & 6.5 & 2.13 & 7.4 & 2.45 & 6.6 & 2.17 & 6.4 & 2.09 & - & - \\
\hline N94 & Peripheral neuritis/neuropathy & - & - & 6.2 & 2.07 & 6.5 & 2.22 & 6.6 & 2.25 & 6.5 & 2.28 & 5.7 & 2.07 \\
\hline U99 & Urinary disease. Other & - & - & 20.4 & 1.22 & 20.3 & 1.24 & - & - & - & - & - & - \\
\hline
\end{tabular}

apeople who stayed in the same cluster throughout the period of study

${ }^{b}$ Prev: prevalence

${ }^{\mathrm{C}} \mathrm{O} / \mathrm{E} \mathrm{r}$ : observed/expected ratio. Italic numbers indicate $\mathrm{O} / \mathrm{E} \mathrm{r} \geq 2$

80 and older, for which the cardiovascular pattern was the highest (Fig. 3).

\section{Discussion}

We explored multimorbidity patterns and their 6-year evolution in people aged 65 years and older with multimorbidity attended in PHC. The most prevalent chronic diseases, Hypertension, uncomplicated and Lipid disorder, were represented in all clusters in all four groups (i.e., men and women aged $65-79$ and $\geq 80$ years). We found 6 clusters per group, 5 of them with a specific pattern related to an organic system: Musculoskeletal, Endocrine-metabolic, Digestive/Digestive-respiratory, Neuropsychiatric and Cardiovascular patterns. We analysed multimorbidity patterns over 6 years and found that they remained quite similar from the beginning to the end of the study period.

We observed a high prevalence of multimorbidity in our population sample, with a higher proportion for women, as in other published studies $[5,8]$ and described 6 patterns in each study group. In addition, the prevalence of chronic diseases and multimorbidity patterns was similar to previous studies in Catalonia [22] and in other developed countries [23-25]. In a separate study in the same sample, we analysed mortality rates and observed higher mortality among men with Digestive-respiratory patterns and among women with Cardiovascular pattern [26]. 


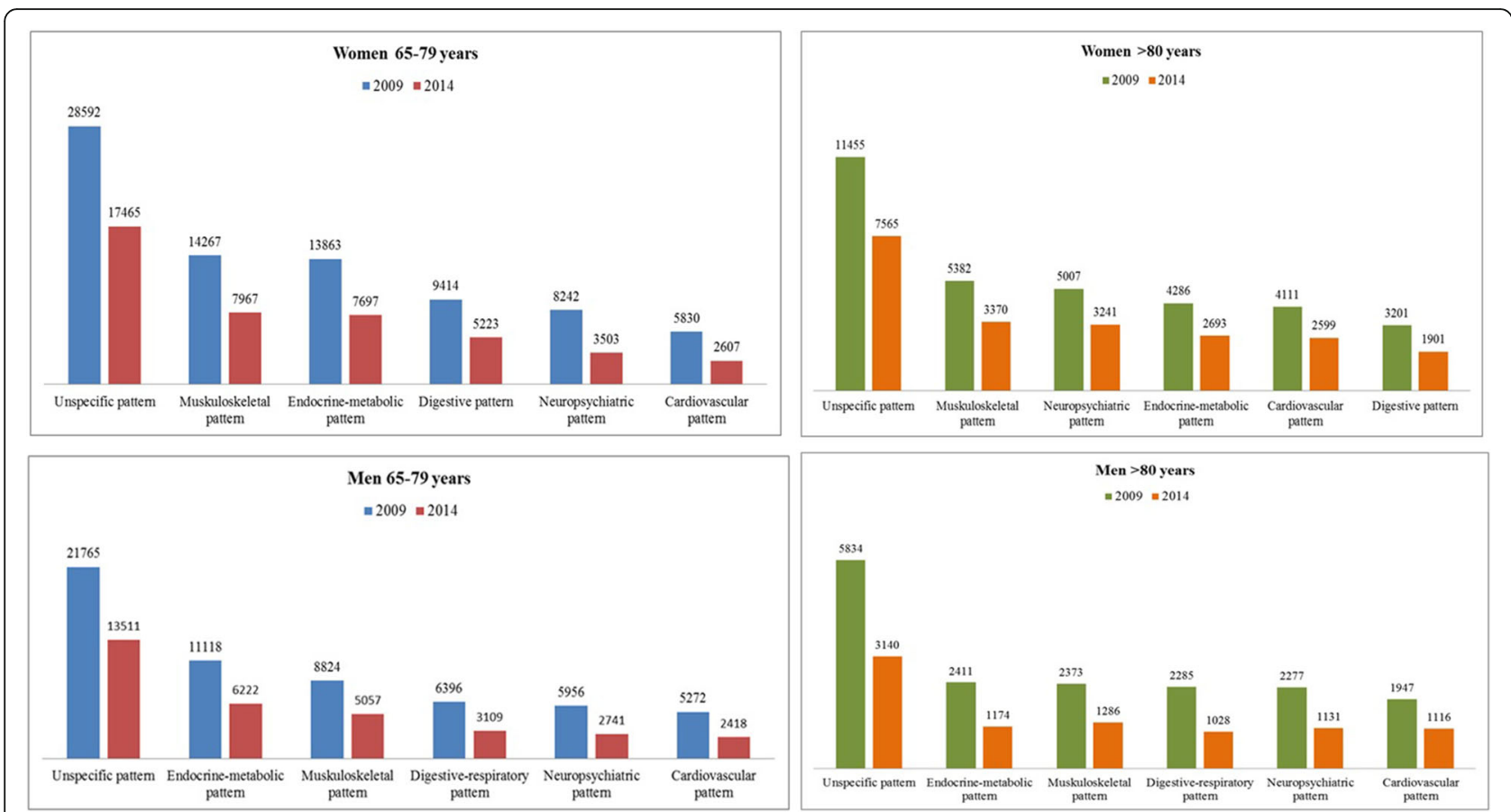

Fig. 3 Sample corresponding to each pattern and people remaining in that pattern at the end of the study

In both age groups, both men and women had the same 5 multimorbidity pattern names plus one additional cluster: a Digestive disease pattern in women and a Digestive-respiratory pattern in men. This difference is probably related to the smoking and alcohol habits that were more common among men than among women in the age groups studied [27]. The differences observed between age groups were related to disease prevalence and $\mathrm{O} / \mathrm{E}$ ratio; no significant differences between men and women were found in the systems that were most commonly affected by the prevalent diseases. As a result, future clinical guidelines could focus on improving common management of multimorbidity in all older patients.

It is particularly noteworthy that more than $50 \%$ of those showing the Nonspecific pattern remained in that same pattern across the period analysed, without moving on to a specific pattern; a few degenerative diseases were added in the older groups. In addition, this first (Nonspecific) cluster was defined by highly prevalent diseases, with no over-represented chronic diseases, so that the association between diseases could exist by chance. Consequently, this first cluster showed that a considerable portion of the sample had no system-specific pattern.

In contrast, across the specific patterns we also observed a large proportion (range from 42.5 to 64.7\%) of people remaining stable (in terms of chronic disease prevalence) in the same pattern. Maximum stability was observed for the Nonspecific pattern in both groups aged 65 to 79 years and in older women; for men aged 80 and older, the Cardiovascular pattern showed the greatest stability. Moreover, some people changed from one pattern to another but the multimorbidity pattern kept mostly stable during the 6 years studied, confirming the long-term stability of the multimorbidity pattern composition. In view of these results, an association could be hypothesized between multimorbidity and specific genetic conditions, as well as previously suggested associations with lifestyle and environmental conditions [28].

Estimates of multimorbidity pattern prevalences differ deeply in the literature because of variations in methods, data sources and structures, populations and diseases studied. Although this makes it challenging to compare study results $[5,29,30]$, there are some similarities between the present and previous studies. For instance, the most common organic systems affected in previous studies of multimorbidity patterns were cardiovascular/metabolic, neuropsychiatric (mental health), and musculoskeletal [30]. Our study found patterns affecting these same organic systems; however, it offers another point of view for defining multimorbidity patterns. Cluster analysis shows the complexity of multimorbidity in persons aged 65 years and older and is likely to be helpful in shaping future strategies to continue studying this important health issue.

Previous studies have analysed no more than four years of data [29], compared to six years of information about the evolution of a multimorbidity pattern in our study. As a result, we identified long-term stability in multimorbidity patterns, observing some differences between age groups, related to prevalence and $\mathrm{O} / \mathrm{E}$ ratio in chronic diseases. Useful information can be extracted from our study for the monitoring and treatment of each multimorbidity pattern. 


\section{Strengths and limitations}

A major strength of this study is the analysis of a large, high-quality EHR database, representative of a large population. In the context of a national health system with universal coverage, EHR data have been shown to yield more reliable and representative conclusions than those derived from survey-based studies [25]. The inclusion of all chronic diagnoses registered in EHR contributed to a more accurate analysis of the multimorbidity patterns in this population. Moreover, the use of data collected by the primary health care system increased the external validation of the information extracted because primary care centres in Barcelona attended more than $70 \%$ of the population at least once a year during the study period. As the nonspecific pattern contained well-known chronic diseases with established clinical guidance, the information extracted is relevant but less useful in clinical practice than the specific patterns defined. The long time period observed provided information on the stability of the patterns during six years, enabling us to focus on creating better strategies to address all five specific patterns in terms of prevention, diagnosis, and treatment of these systemic clusters of prevalent diseases.

A number of limitations must be taken into account as well. First, EHR accuracy depends on the data entered by each general physician or nurse, and EHR systems are not designed as general-purpose research tools [31]. Another weakness could be the attention only to chronic diseases, which precludes awareness of acute diseases or bio-psychosocial factors [2]. Nonetheless, the inclusion of a wide range of diseases makes it possible to find multimorbidity patterns not previously obtained and increases complexity in terms of assembling patterns. Finally, we did not have data on cause of death.

In addition, using MCA can produce low percentages of variation on principal axes, complicating the choice of the number of dimensions to retain. We assumed a fivedimension solution, using the elbow rule in the scree plot to have the most accurate solution possible without including an unwieldy number of dimensions in the analysis [19]. Although we did not retain the total variance of the dataset, clustering techniques can be applied to the reduced dataset while preserving its complexity.

The strength of using k-means cluster analysis is that the results are less susceptible to outliers in the data, the influence of the chosen distance measure, or the inclusion of inappropriate or irrelevant variables. The method can also analyse extremely large data sets (as in this study), as no distance matrix is required. On the other hand, some disadvantages of the method are that different solutions can occur for each set of seed points and there is no guarantee of optimal clustering [11]. To minimize this shortcoming, we tested the internal validity of our solution using bootstrap methods [32], and the results were highly stable (Jaccard $>0.85$ ). However, the method is not efficient when a large number of potential cluster solutions are to be considered [11]; to address this limitation, we computed the optimal number using analytical indexes like Calinski Harabasz [33].

\section{Future research}

With this confirmation of the stability of multimorbidity patterns across age groups, sex, and time, some actions could be considered to improve multimorbidity management. For instance, clinical guidance could encompass a specific pattern to deal with its complexity rather than creating multiple guidelines for each of the chronic diseases. Relevant information could be extracted from our study for the monitoring and treatment of each multimorbidity pattern. Finally, genetic factors, as well as socioeconomic status, should be taken into account in future studies.

\section{Conclusions}

We identified a very large proportion of people over 65 years with multimorbidity, distributed in six clusters; five affected a specific system in the body and one had a nonspecific pattern. The major portion of the sample fit this last pattern, which had few diseases; this finding could be related to genetic or social characteristics of the sample. On the other hand, stability in a specific pattern over an extended time period might give us the information needed to take a new approach and improve a patient's situation. For instance, a new clinical practice guideline could be developed to control a combination of chronic diseases rather than each one individually.

As the prevalence of chronic diseases was stable over the period studied, multimorbidity patterns also became firmer. Therefore, the k-means technique is useful to analyse multimorbidity patterns in real-world data.

The observation that multimorbidity patterns are constant over time is very useful for the specific clinical management of each patient who fits a specific multimorbidity pattern. Further studies using this method in other groups of patients should be performed to validate the results obtained.

\section{Additional files}

Additional file 1: Multimorbidity patterns in women 65-79 years across the period analysed. Patterns defined by prevalence $>20 \%$ and ratio O/ $\mathrm{E}>$ 2. (XLSX $26 \mathrm{~kb})$

Additional file 2: Multimorbidity patterns in men 65-79 years across the period analysed. Patterns defined by prevalence $>20 \%$ and ratio $\mathrm{O} / \mathrm{E}>$ 2. (XLSX $32 \mathrm{~kb})$ 
Additional file 3: Multimorbidity patterns in women aged $>80$ years across the period analysed. Patterns defined by prevalence $>20 \%$ and ratio O/E > 2. (XLSX $29 \mathrm{~kb})$

Additional file 4: Multimorbidity patterns in men $>80$ years across the period analysed. Patterns defined by prevalence $>20 \%$ and ratio $\mathrm{O} / \mathrm{E}>2$. (XLSX $37 \mathrm{~kb})$

\section{Abbreviations}

CHI: Catalan Health Institute; EHR: Electronic health records; ICD10: International Classification of Diseases version 10; ICPC-2: International Classification of Primary Care second edition; IDIAP Jordi Gol: Institut Universitari d'Investigació en Atenció Primària Jordi Gol; IQR: Interquartile range; MCA: Multiple Correspondence Analysis; O/E ratios: Observed/ Expected ratios; PHCs: Primary health care centres; SD: Standard deviation; SIDIAP: Information System for Research in Primary Care

\section{Acknowledgements}

We thank the Catalan Health Institute and the SIDIAP, which provided the database for the study. The authors also appreciate the English language review by Elaine Lilly, PhD, are grateful to Carmen Ibáñez for administrative work and the statistical task by Patricia Garcia.

\section{Funding}

This manuscript constitutes a part of the PhD thesis of MGC in the Public Health Department of the Universitat Autònoma de Barcelona.

This work was supported by a pre-doctoral grant from Catalan Health Institute in Barcelona; by the Catalan Society of General Practitioners (CAMFiC) and by SIDIAP grant to MGC in 2015; this latter organization allowed us to explore thei dataset to obtain the results. The funders had no role in the study design or data collection, analysis, and interpretation, writing of the manuscript, and decision to submit for publication

\section{Availability of data and materials}

The data that support the findings of this study are available from SIDIAP but restrictions apply to the availability of these data, which were used under license for the current study, and so are not publicly available. However, data are available from the authors upon reasonable request and with permission of SIDIAP, as the results from appendix 1-2 corresponding to years 2010-2013.

\section{Authors' contributions}

All authors contributed to the design of the study, revised the article, and approved the final version. MGC, CV, OFB, ARL, TLJ, MAM, and MPV drafted the study protocol and obtained the funding. MGC, CV, ARL, and TL contributed to the analysis and interpretation of data. MGC, CV, QFB, TLJ; $A R L, M A M$, and MPV wrote the first draft, and all authors contributed ideas, interpreted the findings, and reviewed various drafts of the manuscript. All authors read and approved the final manuscript.

\section{Ethics approval and consent to participate}

The protocol of the study was approved by the Committee on the Ethics of Clinical Research, Institut Universitari d'Investigació en Atenció Primària Jordi Gol (IDIAP Jordi Gol) (Protocol No: P15/149). All data were anonymized and the confidentiality of EHR was respected at all times in accordance with national and international law.

\section{Consent for publication}

Not applicable

\section{Competing interests}

The authors declare that they have no competing interests.

\section{Publisher's Note}

Springer Nature remains neutral with regard to jurisdictional claims in published maps and institutional affiliations.

\section{Author details}

'Institut Universitari d'Investigació en Atenció Primària Jordi Gol (IDIAP Jord Gol), Gran Via Corts Catalanes, 587 àtic, 08007 Barcelona, Spain. ${ }^{2}$ Universitat
Autònoma de Barcelona, Campus de la UAB, Plaça Cívica, 08193, Bellaterra (Cerdanyola del Vallès), Barcelona, Spain. ${ }^{3}$ Gerència d'Àmbit d'Atenció Primària Barcelona Ciutat, Institut Català de la Salut, Carrer de Balmes, 22, Barcelona, Spain. ${ }^{4}$ Faculty of Nursing, Universitat de Girona, Emili Grahit, 77 17071 Girona, Spain. ${ }^{5}$ Department of Psychiatry, Hospital Universitari de Vic, Francesc Pla el Vigatà, 1, 08500 Vic, Spain. 'Unitat de Suport a la Recerca, Institut Universitari d'Investigació en Atenció Primària Jordi Gol (IDIAP Jordi Gol), Carrer de Sardenya, 375, Barcelona, Spain.

Received: 20 September 2017 Accepted: 1 January 2018

Published online: 16 January 2018

\section{References}

1. Valderas JM, Sibbald B, Salisbury C. Defi ning Comorbidity: implications for understanding health and health services. Ann Fam Med. 2009;7:357-63.

2. Le Reste JY, Nabbe P, Rivet C, Lygidakis C, Doerr C, Czachowski S, et al. The European general practice research network presents the translations of its comprehensive definition of multimorbidity in family medicine in ten European languages. PLoS One. 2015;10(1):e0115796.

3. Murray CJL, Barber RM, Foreman KJ, Ozgoren AA, Abd-Allah F, Abera SF, et al. Global, regional, and national disability-adjusted life years (DALYs) for 306 diseases and injuries and healthy life expectancy (HALE) for 188 countries, 1990-2013: quantifying the epidemiological transition. Lancet. 2015;386: 1990-2013.

4. Aboderin I, Kalache A, Ben-Shlomo Y, Lynch JW, Yajnik CS, Kuh D, et al. (2002) Life course perspectives on coronary heart disease, stroke and diabetes: key issues and implications for policy and research. Geneva, World Health Organization. http://apps.who.int/iris/bitstream/10665/67174/1/ WHO_NMH_NPH_02.1.pdf Accessed 19 July 2017.

5. Violan C, Foguet-Boreu Q, Flores-Mateo G, Salisbury C, Blom J, Freitag M, et al. Prevalence, determinants and patterns of multimorbidity in primary care: a systematic review of observational studies. PLoS One. 2014;9(7):3-11.

6. Bayliss EA, Ellis JL, Steiner JF. Subjective assessments of comorbidity correlate with quality of life health outcomes: initial validation of a comorbidity assessment instrument. Health Qual Life Outcomes. 2005:3(1):51.

7. Fortin M, Bravo G, Hudon C, Lapointe L, Almirall J, Dubois MF, et al. Relationship between multimorbidity and health-related quality of life of patients in primary care. Qual Life Res. 2006;15(1):83-91.

8. Marengoni A, Angleman S, Melis R, Mangialasche F, Karp A, Garmen A, et al. Aging with multimorbidity: a systematic review of the literature. Ageing Res Rev. 2011;10(4):430-9.

9. Holzer BM, Siebenhuener K, Bopp M, Minder CE. Evidence-based design recommendations for prevalence studies on multimorbidity: improving comparability of estimates. Popul Health Metr. 2017;15(1):9.

10. Everitt BS, Landau S, Leese M, Stahl D. Cluster analysis. 5th ed. Chichester: Wiley; 2011; p. 321-30.

11. Liao M, Li Y, Kianifard F, Obi E, Arcona S. Cluster analysis and its application to healthcare claims data: a study of end-stage renal disease patients who initiated hemodialysis. BMC Nephrol. 2016;17(4):25

12. IImarinen $P$, Tuomisto LE, Niemelä O, Tommola M, Haanpää J, Kankaanranta $\mathrm{H}$. Cluster analysis on longitudinal data of patients with adult-onset asthma. J Allergy Clin Immunol Pract. 2017;5(4):967-78. e3

13. Official numbers of population in Barcelona. http://www.ben.cat/estadistica/ castella/dades/tpob/pad/ine/a2010/edat/edatq01.htm Accessed 19 July 2017.

14. Memòria 2013. Institut Català de la Salut. http://ics.gencat.cat/web/.content/ documents/memoriesICS/ics2013.pdf Accessed 20 Jul 2017.

15. Del Mar G-GM, Hermosilla E, Prieto-Alhambra D, Fina F, Rosell M, Ramos R, et al. Construction and validation of a scoring system for the selection of high-quality data in a Spanish population primary care database (SIDIAP). Inform Prim Care. 2012;19(3):135-45.

16. Prieto-Alhambra D, Judge A, Javaid MK, Cooper C, Diez-Perez A, Arden NK Incidence and risk factors for clinically diagnosed knee, hip and hand osteoarthritis: influences of age, gender and osteoarthritis affecting other joints. Ann Rheum Dis. 2014;73(9):1659-64.

17. Ramos R, Balló E, Marrugat J, Elosua R, Sala J, Grau M, et al. Validity for use in research on vascular diseases of the SIDIAP (information system for the development of research in primary care): the EMMA study. Rev Esp Cardiol (Engl Ed). 2012;65(1):29-37.

18. O'Halloran J, Miller GC, Britt H. Defining chronic conditions for primary care with ICPC-2. Fam Pract. 2004;21(4):381-6. 
19. Sourial N, Wolfson C, Zhu B, Quail J, Fletcher J, Karunananthan S, et al. Correspondence analysis is a useful tool to uncover the relationships among categorical variables. J Clin Epidemiol. 2010;63(6):638-46.

20. García-Gil M, Blanch J, Comas-Cufí M, Daunis-i-Estadella J, Bolíbar B, Martí R, et al. Patterns of statin use and cholesterol goal attainment in a high-risk cardiovascular population: a retrospective study of primary care electronic medical records. J Clin Lipidol. 2016;10(1):134-42.

21. Schäfer I, Kaduszkiewicz H, Wagner H-O, Schön G, Scherer M, van den Bussche H. Reducing complexity: a visualisation of multimorbidity by combining disease clusters and triads. BMC Public Health. 2014;14(1):1285.

22. Foguet-Boreu Q, Violán C, Rodriguez-Blanco T, Roso-Llorach A, Pons-Vigués M, Pujol-Ribera E, et al. Multimorbidity patterns in elderly primary health care patients in a South Mediterranean European region: a cluster analysis. PLoS One. 2015;10(11):1-14.

23. Britt HC, Harrison CM, Miller GC, Knox SA. Prevalence and patterns of multimorbidity in Australia. Med J Aust. 2008;189(2):72-7.

24. Barnett K, Mercer SW, Norbury M, Watt G, Wyke S, Guthrie B. Epidemiology of multimorbidity and implications for health care, research, and medical education: a cross-sectional study. Lancet. 2012;380(9836):37-43.

25. Violán C, Foguet-Boreu Q, Hermosilla-Pérez E, Valderas JM, Bolíbar B, Fàbregas-Escurriola $\mathrm{M}$, et al. Comparison of the information provided by electronic health records data and a population health survey to estimate prevalence of selected health conditions and multimorbidity. BMC Public Health. 2013:13:251.

26. Ibarra-Castillo C, Guisado-Clavero M, Violan Fors C, Pons-Vigués M, López-Jiménez T, Roso-Llorach A. Survival in relation to multimorbidity patterns in older adults in primary care in Barcelona, Spain (2010-2014): A longitudinal study based on electronic health records. J Epidemiol Community Health. (in press).

27. Lopez AD, Collishaw NE, Piha T. A descriptive model of the cigarette epidemic in developed countries. Tob Control. 1994;3(3):242-7.

28. Hu JX, Thomas CE, Brunak S. Network biology concepts in complex disease comorbidities. Nat Rev Genet. 2016;17(10):615-29.

29. France EF, Wyke S, Gunn JM, Mair FS, McLean G, Mercer SW. Multimorbidity in primary care: a systematic review of prospective cohort studies. $\mathrm{Br} J \mathrm{Gen}$ Pract. 2012;62(597):e297-307.

30. Prados-Torres A, Calderón-Larrañaga A, Hancco-Saavedra J, Poblador-Plou B, Van Den Akker M. Multimorbidity patterns: a systematic review. J Clin Epidemiol. 2014;67(3):254-66.

31. Coorevits P, Sundgren M, Klein GO, Bahr A, Claerhout B, Daniel C, et al. Electronic health records: new opportunities for clinical research. J Intern Med. 2013;274(6):547-60.

32. Hennig C. Cluster-wise assessment of cluster stability. Comput Stat Data Anal. 2007:52(1):258-71.

33. Caliński T, Harabasz J. A dendrite method for cluster analysis. Commun Stat Methods. 2007:3(1):1-27.

\section{Submit your next manuscript to BioMed Central and we will help you at every step:}

- We accept pre-submission inquiries

- Our selector tool helps you to find the most relevant journal

- We provide round the clock customer support

- Convenient online submission

- Thorough peer review

- Inclusion in PubMed and all major indexing services

- Maximum visibility for your research

Submit your manuscript at www.biomedcentral.com/submit
Biomed Central 